\title{
"A te convien tenere altro viaggio": il problema della auctoritas e il genere della Commedia
}

Nel Convivio (4.6.3-5) Dante definisce "autoritade" come "atto d'autore" e dà due accezioni del termine "autore": la prima deriva da "aueio" e significa "legare parole", la seconda da "autentin" e significa "degno di fede e di obbedienza". Le due accezioni appartengono a due sfere d'azione distinte, rispettivamente la poesia e la filosofia, e della prima, Dante aggiunge, "al presente non s'intende". Tuttavia l'accostamento actor auctor autentin non è certo casuale e Dante se ne serve per ridefinire la propria auctoritas: assumendo i ruoli di autore e commentatore Dante li personalizza, e presentando i testi poetici come degni di commento, il quale, si badi, rimane loro 'servo' (1.5.6), conferisce loro una

unprecedented auctoritas for 'modern' works and expresses his specific desire to excercise a control over our understanding of them, which is ... denied in practice if not in theory to the ancient auctores. [...] The three books that follow the introduction continue to be profoundly concerned with constructing an authority-conferring ethicalintellectual history of the self. (Ascoli 34-35)

In altre parole, la distinzione tra poesia e filosofia rimane ma, poiché è possibile che un poeta sia anche filosofo, Dante, tramite il procedimento autobiografico, si conferisce la auctoritas di entrambe. Nella Commedia, abbandonato il sussidio del commento in prosa, è alla struttura del poema che viene affidato il compito di rivelare l'intenzionalità dell'autore; il tema del controllo autoriale del testo e della sua corretta lettura si articola e sviluppa tramite l'assunzione, a soggetto dell'opera, della reinterpretazione della storia dell'umanità e dei suoi testi. I riferimenti ai libri, all'atto del leggere sono così numerusi nella Commedia che il Mazzotta l'ha definita "a book about books" (151), tanto da poterla interpretare anche come un'allegoria della stessa lettura e conseguente-mente della funzione della scrittura.

Se ogni [ri]lettura e ogni [ri]scrittura sono necessariamente [re]interpretazioni che modificano il libro della memoria, allora non si puo sfuggire all'allegoria, intesa nel senso generico di dipendenza dell'interpretazione di un testo dall'ottica personale e culturalmente connotata di chi interpreta, in 
quanto l'ottica impone a quel testo significati non previsti dall'autore e magari alieni alla cultura cui autore e testo appartengono. Dante, nell'assumere la storia e la scrittura umana a soggetto della esplorazione del vero, si pone il problema del come arrivare, tra le tante possibilità di interpretazione, a quella corretta; il che vuol dire chiedersi se l'uomo ha i mezzi sufficienti a conoscere la verità. Per il critico, d'altro canto, si pone il problema di individuare quale genere di allegoria, quella dei poeti o quella dei teologi, sia applicabile alla Commedia, e dunque quale rapporto ci sia tra il metodo di scrittura adottato in quest'opera rispetto a quelli della Vita Nuova e del Convivio. Se e lecito applicare la lettura allegorica a qualsiasi testo letterario, alla storia, al mito, nasce il problema parallelo, relativo alla produzione del testo, della intenzionalità dell'autore: come giustificare, da una parte, un'interpretazione chiaramente non prevista dall'autore (è il caso di Virgilio), e come, dall'altra, costruire un'opera in modo che il significato inteso dall'autore non sia equivocabile, data la ambiguità inerente alla poesia.

Lo scopo di questo lavoro è di capire quali risposte Dante dia a questi quesiti, e, in conseguenza, di definire i principi poetici che informano la Commedia.

Prima di cominciare però, va chiarita quella che può essere l'ottica di Dante. Credo che il problema dell'allegoria vada impostato diversamente: nei riguardi della Commedia la distinzione tra allegoria dei poeti e allegoria dei teologi, con tutte le complicazioni che questo comporta, è fuorviante. Mi sembra più oppor-tuno e più utile distinguere invece tra l'interpretazione allegorica nel senso generico summenzionato e l'allegoria di carattere specificamente cristiano, che nasce cioe dall'ottica cristiana. In altre parole la distinzione che mi sembra più corretta, e che più si attaglia alla problematica dantesca, non è quella tra poeti e teologi bensì tra poeti cristiani e non. Mi spiego. L'avvento del cristianesimo provoca una profonda rottura ideologica sia col mondo giudaico che con quello ellenico, e una drastica deviazione nel corso della storia: un nuovo anno zero che separa tutta la storia precedente alla venuta di Cristo dalla nuova epoca evangelica, la quale però non può, nel suo divenire, non fare i conti col passato e, per questo, incorrere in difficili problemi da risolvere, e in particolare questi: cosa fare di tutta la sapienza antica? E di tutti gli uomini giusti del passato? Il Verbo di Dio non era forse presente nel mondo anche prima che si incarnasse in Cristo? Dal momento che si vuole rispondere a queste domande "Christianity accepts the responsibility for the whole preceding history of humanity; but then it also claims the benefit. All the evil that is done is done against the Word, but since, conversely, all the good that is done is done by help of the Word, who is the Christ, all truth is as by definition, Christian" (Gilson 27).

E da questo punto che nasce l'allegoria cristiana: tutto il bene e tutta la verità che si può trovare nel mondo pre-cristiano diventano prefigurazioni 
della rivelazione salvifica di Cristo, per cui non c’è nessuna contraddizione logica nell'interpretare la quarta Ecloga virgiliana come profezia del cristianesimo, o nel definire cristiani ante litteram alcuni pagani, e salvarli. La concezione monoteistica cristiana presenta il vantaggio di poter ripristinare la continuità storica, e di poterne usufruire, poiché tutta la storia è parte del disegno provvidenziale divino, il cui unico scopo è la salvezza dell'umanità. Inoltre la concezione di un Dio creatore e onnisciente offre il vantaggio di poter risolvere i problemi epistemologici che la filosofia lasciava aperti. Il problema della conoscenza viene risolto metafisicamente: la verità è al di sopra della incerta transitorietà del mondo reale; è oggettiva, assoluta, creata da Dio, e l'oggetto della conoscenza che si presenta all'intelletto è un ritorno al punto di origine, Dio. La conoscenza perde così ogni valore intrinseco; il Verbo ci rivela quanto e non più di quanto ci serve alla nostra salvezza; tutto il resto è curiosità vana. Se da una parte tutto questo finisce col mettere il bastone tra le ruote al progresso della scienza e delle arti (per la diffidenza che ne deriva nei confronti della dialettica, della retorica e delle immagini sacre, e per il posto infimo cui è relegata la musica strumentale), dall'altra il carattere e il successo della rivoluzione apportata dal cristianesimo non possono essere semplicisticamente delimitati dalla sostituzione della ragione con la fede. Le due cose, pur essendo ben distinte, non sono mai in realtà separate nel pensiero cristiano (tanto più che tutti i testi sono, al di fuori del Vangelo, non cristiani); la fede non ha bisogno di prove e, allorquando le verità della ragione coincidono con quelle della fede, è la fede che convalida quelle e non viceversa, ma allo stesso tempo viene affermata la "razionalità" della fede. Non pud esserci una rinuncia alla razionalità se la teologia si assume il compito di elaborare una metafisica della conoscenza, diventando così essa stessa scienza e avvalendosi perciò, pur sempre distinguendosene, di alcuni dei mezzi propri della filosofia.

È dunque entro le complessità e spesso paradossalità di questo contesto che si definisce, e non facilmente, la posizione di Dante nei riguardi del rapporto tra fede e ragione, tra teologia e filosofia, e soprattutto nei riguardi della funzione dell'arte, perché per uno che, come Dante, è squisitamente poeta e profonda-mente cristiano, questi importanti quesiti confluiscono in quello sulla possibilità e la natura di una poesia "Cristiana".

\section{1) Poesia e teologia}

La Commedia si apre col ben noto topos dello smarrimento nella selva e della quête dell'eroe: uscito dalla dimensione del sonno, della non-consapevolezza, Dante si ritrova nella selva selvaggia a cercare la "dritta via". Quel "ritrovarsi" ha due significati: 1) ritrovare letteralmente se stesso, tornare ciod alla 
piena coscienza di sé, della propria situazione e quindi alla propria libertà e responsabilità morale, e 2) trovarsi "di nuovo" nella posizione di dover scegliere un percorso cognitivo e poetico. ${ }^{1}$ Qui il viaggio sarà "altro" da quello tentato in precedenza nella Vita Nuova e nel Convivio, e in che modo il primo fosse diverso Dante ce lo spiega subito: si trattava di un viaggio incontro al sole "che mena dritto altrui per ogne calle", del tentativo, ora scartato, di salire direttamente sul colle illuminato. La "verace via" si è rivelata non così diretta come aveva sperato, la rinuncia al male (è già salvo dai pericoli del "pelago") e il desiderio del bene non bastano per salire perché per liberarsi veramente dall'errore è necessario capirne la natura, le cause, gli effetti. E imparare a distinguerlo di volta in volta dalla verità è impresa molto più complicata di quanto non avesse previsto perché esso può celarsi dietro un'apparente, o parziale, verità, così come la verità può avere talora anche "faccia di menzogna". Insomma Dante, ricacciato nella solitudine e nel silenzio del "gran diserto", riprende la sua quête da capo, dal silenzio della Vita Nuova che si era chiusa con una visione di un bene ineffabile che avrebbe dovuto costituire la nuova materia poetica, e con la rinucia alla scrittura, non avendo ancora trovato il modo di trattare una così alta materia. Il modo diverso di cui ora si avvale è questo: "ma per trattar del ben ch'i'vi trovai, / diro de l'altre cose ch'io v'ho scorte"; non presenterà solo l'ideale di virtù (V.N.) e di sapienza (Conv.), ma anche il suo contrario, non gli astratti concetti ma il concreto della storia umana, di una complessa realtà in cui si intrecciano modi e gradi diversi di estraniazione e di approssimazione all'ideale di bene e di felicità, nella cui attuazione si realizza la natura e il destino dell'essere umano.

In questo "altro viaggio" dunque la selva è inevitabile perché la selva rappresenta la vita umana, ${ }^{2}$ e percio la dritta via non è quella che lo conduce fuori da essa ma ad addentrarsi in essa per capirne la realtà del bene e del male che vi si confondono. La Commedia è allora anche una metafora della stessa quête: la ricerca della verità attraverso l'analisi delle possibilità che i mezzi umani di conoscenza hanno di trovarla. La fictio del viaggio fisico che, attraverso l'Inferno e il Purgatorio, lo porta nel luogo assegnato da Dio all'uomo perché vi vivesse felice, rappresenta un viaggio a ritroso nella storia verso un tempo che gli antichi chiamarono l'età dell'oro, per scoprire come la divina foresta spessa e viva si è trasformata in una selva selvaggia e aspra e forte, e come l'uomo può ricongiungersi col proprio destino. L'elemento unificatore è nella Commedia, come nella Vita Nuova, la memoria, con la differenza fondamentale che qui si tratta di memoria collettiva, la storia appunto; e come guida alla lettura di questo "libro della memoria" Dante sceglie Virgilio (anzi è la Beatrice che è opera di fede a sceglierlo per lui, in virtù della sua parola ornata e onesta). Virgilio è "maestro" e "autore", esempio massimo di poesia e di sapienza, e quindi anche prova del fuoco 
dell'unione delle due cose, ed anche giustificazione della missione salvifica che Dante affida alla pocsia. Ma se Virgilio deve rappresentare per Dante una fonte di salvezza oltre che di poesia, così come lo era stato per Stazio ("Per te poeta fui, per te cristiano"; Purg. 22.73), Dante deve affrontare il problema della difesa di quella lettura allegorica dei versi della quarta Ecloga, interpretati come profezia dell'avvento del cristanesimo, che sant'Agostino aveva respinto e che Virgilio non aveva certo previsto: se così fosse egli sarebbe salvo. E Dante, volendo, avrebbe potuto salvarlo, come ha fatto con altri pagani, ma preferisce presentarcelo come colui che illumina gli altri e non se stesso, perché è in questo ruolo di segno conflittuale nella ricerca della verità che gli serve. Scegliere il Virgilio storico, escluso dalla grazia divina, vuole dire dimostrare che la verità non è appannaggio esclusivo dei cristiani e che la ricerca della verità è un dovere che continua, e che continua ad essere difficile in questa selva erronea della vita, senza la guida di un papa e di un monarca che riescano a vedere, della vera citta, almeno la torre. Vuol dire valorizzare e allo stesso tempo mettere alla prova le capacità e i limiti della ragione umana. Vuol dire infine mettere alla prova la validita della memoria come via per risalire a un ideale di civilta che approssima l'ordine divino. Lo scopo del viaggio a ritroso che la memoria permette di intraprendere è trovare un filo con-duttore che percorra e dia un significato e uno scopo alla storia tutta: il ricordo cristiano e quello pagano di un'era aurea di civilta sono un'unica verità e un unico ideale di perfezione terrena cui l'umanità aspira e che dovrebbe forse essere ancora possibile.

Attraverso la figura di Virgilio viene esplorato il rapporto tra letteratura e storia, tra retorica e verita, con tutte le difficoltà che questo implica: se la retorica è insieme costruzione e ricostruzione della storia, in che cosa consiste la credibilità della retorica e della letteratura in particolare? E se essa è l'unico modo che abbiamo di ricomporre il "libro della memoria", che validità può avere l'assumere la storia come "lettera"? In altre parole, se ogni lettura è interpretazione, che cosa garantisce che ci sia, tra le tante, un'interpretazione corretta?

Tradizionalmente Virgilio è stato visto come simbolo della ragione umana o della filosofia, e/o come rappresentante di Roma (Nardi 165, 275; Comparetti 180-81; Mastrobuono 89). La condanna di Virgilio e degli spiriti magni al Limbo è attribuita ai limiti della ragione umana non sorretta dalla fede; Virgilio infatti la spiega non solo nei termini che ci aspetteremmo, e cioè per non aver conosciuto Dio e non per aver peccato, ma anche di "ribellione". Come ci si può ribellare a qualcosa o qualcuno di cui non si conosce l'esistenza? È naturale dedurre che per Dante deve essere possibile che si arrivi a conoscere Dio pur senza conoscere Cristo, ${ }^{3}$ che la ragione umana possa, nel riconoscere i propri limiti, tradursi in fede. Dico "fede" e non "desiderio" perché la condanna di Virgilio e degli altri spiriti magni consiste 
proprio nell'essere "sospesi" in uno stato di desiderio senza speranza. La speranza è cio che traduce il desiderio in fede proiettandolo verso il futuro, cioè, come dice Mastrobuono (73), la visione pagana (dell'età dell'oro) è relativa al passato, mentre quella giudaica al futuro: mentre gli ebrei vivevano nella speranza, i pagani vivevano nella nostalgia. Ma cosa dire allora della profezia pagana del ritorno dell'età dell'oro? La nostalgia del Parnaso per i pagani e dell'Eden per i cristiani, è comune ricordo di tutti gli uomini, e diventa comune ideale di civilitas, di giustizia terrena. In questo senso la profezia virgiliana può anche essere consapevole; la differenza fondamentale è che essa si limita al comune ideale di civilitas, incapace di fare il passo avanti verso il destino celeste dell'uomo. Questo restare "sospesi" alle soglie della verità dovrebbe spiegare perché non si siano salvati neppure Platone e Aristotele, sulla cui filosofia si basa la teologia cristiana. Chiedersi perché un peccatore cristiano si salvi per una "lacrimetta" mentre Virgilio no, è domanda che nasce dal sentimento che non riesce ad accettare una giustizia così ferrea da sembrare crudele; e certo Dante stesso se la pone senza potervi dare risposta: l'uomo non può pretendere di capire Dio, sa però di potere confidare nella sua giustizia e misericordia, e che la preghiera non è vana (Par. 19.70-90). Nondimeno Dante cerca e trova la logica di questa giustizia all'interno dell'animo umano: a Virgilio contrappone altri due magnanimi pagani, Ulisse e Catone.

Secondo Nardi (Dante 131) Ulisse rappresenta, come Virgilio, la ragione umana, con la differenza che Ulisse si rifiuta di riconoscerne i limiti ed è per questa sua ribellione al decreto divino che è condannato, contrariamente alla tesi di Fubini (1-36) il quale, ricordando che Ulisse si trova nell'Inferno per il peccato di frode e non per il suo, sia pure folle, volo, vede in Dante solo ammirazione per lui. Dirò subito che è cosa ovvia che la frode è la causa della condanna di Ulisse e questo fatto non va dimenticato, e che non c'è dubbio che Dante prova ammirazione per lui; resta comunque il fatto che il suo volo è folle, e resta quindi la necessità di capire il rapporto tra questa follia e il peccato di frode. È folle perché Ulisse crede di avere ali e ha solo remi; solo la grazia divina può tramutare i remi in ali ed e per questo che l' "altrui" che Ulisse troppo tardi riconosce gli nega l'approdo. D'altra parte però è anche vero che l'uomo pud coi soli remi arrivare in vista del monte del Purgatorio, ed è significativo che Dante stesso, avendo seguito la medesima rotta, una volta arrivato ai piedi del monte, miracolosamente scampato ai pericoli del pelago che era stato fatale ad Ulisse, si fosse imbattuto anch'egli nel divieto. L' "altro viaggio", che condivide le motivazioni dell'ultimo viaggio di Ulisse, conoscere gli umani vizi e le virtù, ha come fine il capire come ci si guadagna l'accesso. Il desiderio di conoscenza di Ulisse è desiderio naturale e nobile e non è certo per questo che egli è punito, ma l'essere inghiottito dai flutti e la contrapposizione col carro di Elia (Inf. 26.35) contraddicono la descrizione del viaggio come "volo", perché in lui il desiderio di conoscenza è fine a se stesso, vano, e così l' "orazion picciola" che esprime ideali nobilissimi dimo- 
stra i suoi limiti e si rivela, in ultima analisi, anch'essa fraudolenta. Il desiderio di conoscenza di Ulisse risulta non solo vano ma falso (e qui è la differenza fondamentale tra lui e Virgilio) per la profonda contraddizione che c'è tra di esso e la frode la quale è il suo esatto contrario: nascondere, travisare la verità che egli dice di amare.

Il desiderio di verità di Virgilio è invece genuino e non è macchiato da alcuna perversione d'animo. Egli dimostra anche di riconoscere i limiti della ragione, rimandando Dante costantemente a Beatrice, che è opera di fede, per ottenere risposte che lui non sa dare o non sa dare pienamente. Che cosa lo trattiene allora al di qua dalla fede? Per capirlo, Dante porta sulla scena Catone. Quando Dante aveva espresso il dubbio di non essere degno d'intraprendere il viaggio (Inf. 2), Virgilio lo aveva rassicurato dicendogli che tre donne del Paradiso avevano intercesso per lui; ma adesso, davanti a Catone, un pagano che egli crede vicino al proprio mondo, alla propria cultura, alle donne del cielo aggiunge Marzia, ${ }^{4}$ rivelandosi così incapace di distinguere tra argomenti validi e non per un cristiano. L'impedimento è dunque un habitus che lo lega ai valori di una cultura inevitabilmente diversa da quella cristiana. Catone allora, raggiungendo in atto quello che in Virgilio è in potenza, rappresenta la possibilità concreta che hanno la ragione e $\mathrm{i}$ più alti valori della cultura pagana di valicare i confini cronologici e tradurre l'ideale temporale di civilitas in quello eterno della patria celeste. In questo senso si delimita il concetto della "romanita" di Cristo: Catone che si ribella all'impero romano depositario di un fine provvidenziale, e che col suo sacrificio viene ad assumere "la funzione di figura laica di Cristo" (Barberi-Squarotti 135), rappresenta un ideale di civilitas universale, e di cui Roma non è che un esempio, e una possibilità di salvezza che va al di là della contingenza storica: l'espansione dell'impero romano era stata necessaria alla venuta di Cristo e la venuta di Cristo alla salvazione dell'umanità, ma né l'una né l'altra prescindono dalla capacità e diretta responsabilità dell'individuo nella propria salvezza spirituale.

La previsione di questa possibilità fa parte del problema che Dante si pone di come leggere la storia: se guardando la storia dell'impero romano con l'ottica dell'eterno vi si vede la provvidenzialità divina, quando la si guarda dall'interno e nel tempo, i singoli avvenimenti e personaggi vanno giudicati eticamente per quello che sono, altrimenti il fine provvidenziale verrebbe a giustificare anche tutti gli sbagli e le atrocità, e inoltre va ricordato che $\mathrm{i}$ personaggi storici non sono consapevoli di essere attori di un disegno divino. Una scrittura corretta della storia deve allora tenere conto dell'eterno e del tempo, dell'interpretazione che lega la macrostoria in un libro della memoria e dei giudizi di cui è passibile la microstoria.

Il testo di Virgilio rappresenta un genere simile di scrittura, poiché il viaggio di Enea è visto come necessario, provvidenziale, e perché i fatti narrati sono storicamente veri. I critici concordano nel ritenere che Dante 
prendesse alla lettera il racconto dell'Eneide, tuttavia l'Eneide è anche opera poetica e crea il problema del rapporto tra letteratura e storia. Il metodo che Dante adotta per esplorare questo rapporto è esemplificato dal trattamento del mito: per esempio, il mito di Orfeo è addotto nel Convivio (2.1.3) ad esempio di "fabula" inventata per dire qualcos'altro; allo stesso tempo pero viene sostenuta la autenticità storica del personaggio di Orfeo, che è collocato nel Limbo (Inf. 4.144). La stessa cosa avviene delle figure mitologiche (Caronte, Minosse, i giganti) la cui storicità è confermata dalla somiglianza tra i miti pagani e quelli ebraici. Questo procedimento è costante in tutta la Commedia; Dante non solo cerca nel mito una verità nascosta, ma ridimensiona il mito restituendogli una realtà che nelle favole dei poeti s'era in parte perduta. Particolarmente significativo si presenta sotto questo aspetto il XXX Canto dell'Inferno, dove il tema del falso è inestricabilmente legato alla lingua, ai mezzi che ha la poesia di rappresentare i diversi aspetti del reale, in ordine non tanto alla efficacia quanto alla funzionalità rispetto a finalità didattiche.

La lunga similitudine con cui il canto si apre è in netto contrasto sia per stile che per i personaggi lì evocati, con la rappresentazione della pena dei falsari che segue. Le immagini raccapriccianti, i vocaboli plebei e l'asprezza delle rime fanno sì che la realtà del peccato e della pena risulti al lettore ben vicina e visibile in modo da renderlo direttamente partecipe dell'orrore e della violenza. Violento è anche il distacco da questa visione. Dante ne esce dopo il rimprovero che gli fa Virgilio di avervi troppo partecipato "ad ascoltarli er'io del tutto fiso" (130); ma è da notare come anche Virgilio partecipi di questa violenza; egli pronuncia infatti parole che non ci si aspetta da lui: "or pur mira, / che per poco che teco non mi risso" (131-32). Difatti la violenza opera sulla lingua in tutto il canto e a più livelli. Le inmagini animalesche messe in risalto dal linguaggio e suoni plebei fanno sì che il lettore torni ai versi iniziali per rendersi conto della natura della similitudine: la lingua e tono solenni da una parte riflettono il distacco temporale (l'antichità del mito rispetto al presente Inferno), nonché lo stile delle fonti cui l'autore attinge; d'altra parte fungono da contraddizione all'enunciato (la realtà ugualmente animalesca delle furie tebane e troiane). È da notare inoltre che non è presente solo la contrapposizione tra il tragico e l'umile ma anche la mescolanza dei due; il linguaggio di Mastro Adamo è infatti generalmente elevato, e non senza momenti lirici, malgrado la situazione che lo contraddice.

La Frankel vede in questo episodio un'aperta polemica con Virgilio quale

the representative par excellence of alta tragedia and, hence, as the natural adversary and authoritative antagonist of the product he is witnessing at first hand, the foremost obstacle that Dante has to surmount in his novel enterprise. In a way, Dante is directly confronting Virgil both by exposing him to his dizzying interchange of styles and, even more, by using one of Virgil's own creatures to be the interlocutor of a tenzone with a near-contemporary of Dante in Dante's own territory. No wonder Virgil is both per- 
turbed and angered. [...] I believe that the rite of passage taking place in Inferno 30 has to do with Dante's emancipation from Virgil in the matter of style and language.

[...] Inferno 30 is Dante's battlecry from the tyranny of classical examples and of the rhetoricians' rules. It is Dante's manifesto for his revolutionary 'new style'. (187, 191, 194)

È vero che per Dante l'adozione del modello implica, qui come altrove, il raffronto e l'emancipazione da esso. Il linguaggio tragico inteso come determinante lessicale e come sostenuta solennità tonale non può adattarsi alla esplorazione dei diversi livelli di realtà presenti nell'opera, tuttavia l'episodio presenta non tanto una diretta polemica con Virgilio, quanto il coinvolgere il poeta nel problema della proporzionalità tra l'efficacia poetica e quella etica della rappresentazione: da una parte il linguaggio aulico crea un distacco dalla materia rappresentata, che potrebbe costituire un ostacolo alla sua corretta interpretazione, dall'altra il linguaggio plebeo, presentando da vicino l'orribile scena nella sua più cruda realtà, presenta anch'esso i suoi pericoli, avendo anch'esso un fascino perverso. Insomma l'ottica del troppo vicino è estrema e potenzialmente fuorviante quanto quella del troppo lontano, come le lenti ai due poli di un canocchiale.

Ed è una riflessione sul potere e sulla funzione della metafora: Atamante nella furia insana della caccia, finisce col trasformare con l'immaginazione la sua "preda" in animali braccati; Ecuba nella furia del suo dolore, pur non trasformandosi letteralmente in cagna, "latra" come se lo fosse; la pena che affligge i falsificatori non è anch'essa altro che una metafora di umanità rinnegata e stravolta dal falso. Ma è nella metafora che si vede il significato dei fatti, il perché certe azioni contraddicono e cancellano tutto quello che costituisce l'essere umano. Il pericolo è nel troppo compiacersi del racconto, nel non saper ristabilire la misura. Nel caso di Mastro Adamo è l'individuo che volutamente stravolge la realtà, e Dante, oltre alla difficoltà di dimostrare come ci si impiglia e come ci si slega dalla falsa retorica, crea per sé (e non per Virgilio) un'altra difficoltà; quella di discernere fino a che punto è lecito l'oltraggio alla lingua poetica (lingua ordinata, armonica) per poter descrivere la realta senza travisarla. In questo Virgilio non rappresenta solo tensione ma anche misura:

\section{E fa ragion ch'io ti sia sempre allato, se più avvien che fortuna t'accoglia dove sien genti in simigliante piato: ché voler ciò udire è bassa voglia}

(Inf. 30.145-48).

È una misura che, nella perpetua tensione tra ideale e realtà in poesia, è necessaria a mantenere quel difficile e precario equilibrio che permette di esprimere una realtà (quella infernale) che è negazione della poesia, rendendo 
lecita, anzi necessaria, la creazione di una poesia della negazione, la quale però dalla negazione riporti all'ideale, alla verità.

La retorica ha i suoi limiti e i suoi tranelli, ma è l'unico mezzo di comunicazione e di persuasione che abbiamo per il male come per il bene. Mazzotta, riflettendo sulla inadeguatezza della ragione e della retorica di arrivare alla verità, conclude che Dante ripudia la retorica di Virgilio, essendo l'opposto della lingua "soave e piana" di Beatrice, per cui l'allusione alla parola "ornata" di Virgilio è, secondo lui, ironica $(83,88,157)$.

Però la parola di Virgilio non è solo "ornata" ma anche "onesta", e la retorica di Beatrice, diversa quanto si voglia da quella di Virgilio, è pur sempre retorica. La Commedia d̀ opera di fede e, inadeguatezza a parte, Dante non è certo un agnostico. ${ }^{6}$ Piuttosto bisognerebbe rassegnarsi al fatto che Dante ci presenta i problemi e le difficoltà insite nell'atto dello scrivere, senza darcene necessariamente una risposta univoca o esplicita, o, meglio, la sua risposta è da trovarsi nell'azione, nel fatto che, malgrado tutto, continua a scrivere aprendoci ai suoi dubbi e alle sue certezze per cento canti. Perfino nell'ultimo, che d̀ un canto di dichiarata sconfitta non solo della lingua ma anche della fantasia (cfr. v. 142), non si arrende, e cerca di capire e di dire, secondo le proprie capacità, quel poco che d̀ dato all'uomo di capire e comunicare. Per Dante è assurdo pensare che Dio abbia dato all'uomo l'uso della ragione e il desiderio di conoscenza invano; in altre parole, il desiderio di conoscenza prova che la conoscenza è possibile.

I padri della Chiesa avevano dovuto, volenti o nolenti, piegarsi alla necessità della retorica, e, per difendere la dottrina, avevano dovuto imparare, con i dovuti avvedimenti, dai loro "nemici" pagani, finendo così per assimilarne la cultura trasformandola in termini cristiani e facendo, allegoricamente, dei suoi valori più alti, prefigurazione, e insieme conferma, di verità cristiane. La fede aveva bisogno del sostegno della ragione, e l'uso della retorica veniva giustificato in ordine alla finalità della propagazione della fede (Comparetti 50-118; Schlosser-Magnino 72-80; Dronke 7-38).

Nei trattati di retorica abbondavano in particolar modo gli esempi tratti dalle opere virgiliane; allo stesso tempo sia le opere che il personaggio di Virgilio venivano allegoricamente trasformati a significare qualcosa di più $o$ di diverso da quelle che potevano essere state le intenzioni originali; il viaggio di Enea veniva a rappresentare le età dell'uomo e Virgilio il profeta del cristianesimo?

Il Virgilio dantesco è probabilmente il più vicino al personaggio storico, perlomeno nelle sue vesti di poeta; bisogna chiederci se Dante condividesse l'interpretazione della profezia e, nel qual caso, come risolvesse il problema della intenzionalità. Certamente Dante non credeva che Virgilio avesse previsto l'avvento del cristianesimo (altrimenti, come s'è già detto, sarebbe salvo). Ciò non toglie che si trattava comunque di profezia. L'ideale di pace 
e giustizia che avrebbe ristabilito il ritorno del regno di Saturno coincideva con quello cristiano, ed è per questa "coincidenza" che Virgilio diviene mezzo di salvezza per Stazio il quale per primo "converte" le parole del suo amato autore a significare il messaggio del cristianesimo appena nato. Virgilio aveva insomma intenzionalmente profetizzato una verita in cui credeva, senza però rendersi conto del contenuto effettivo della sua profezia. Tuttavia quello della intenzionalità rimane ugualmente per Dante un problema da risolvere nell'ambito della scrittura: se la verità è al di fuori degli avvenimenti della storia e delle intenzioni e consapevolezza dei suoi attori, l'approccio alla realtà storica come lettera non può essere diverso da quello applicabile alla lettera di un'opera poetica, in quanto alla verita si arriva solo attraverso l'interpretazione. Tutto questo porterebbe allora alla conclusione che il senso letterale di un'opera, e quindi della Commedia, è inevitabilmente diverso da quello inteso dai teologi nei riguardi della Bibbia, e che percio Dante rimane fedele alle formulazioni del Convivio. Tuttavia il metodo adottato nella scrittura della Commedia è, per quanto riguarda la nozione del "soggetto", diverso da quello del Convivio.

Il Convivio era inteso come un tentativo di riscrittura che doveva giovare a quello precedente costituito dalla Vita Nuova, ma l'esperimento del Convivio, come tentativo di sintesi e conciliazione attraverso l'interpretazione allegorica, fallisce per la limitatezza della nozione di allegoria ivi assunta.

Tale procedimento genera confusione riguardo quello che Beatrice deve rappresentare in rapporto alla donna gentile una volta che su quest'ultima viene imposto il ruolo di personificazione della filosofia, e crea una discrepanza insormontabile tra lettera e allegoria, tra poesia e prosa, e finisce col produrre un capovolgimento poiché la "favola", le "belle menzogne" dei poeti, sono create dalla prosa che impone sulla poesia significati ed intenzioni che le sono estranei. La confusione nasce dal voler cancellare la realtà fattuale del soggetto: mentre nella Vita Nuova Dante si peritava di dimostrare che Beatrice, la donna gentile e tutte le altre donne erano persone in carne ed ossa, nel Convivio in essenza lo nega, proponendo che quelle poesie fossero state concepite come allegorie; quello che era vero nella Vita Nuova diventa favola nel Convivio.

Mentre Hollander ammette che nel Convivio la nozione di allegoria è confusa (Allegory 33), ${ }^{8}$ Tateo crede che le canzoni ivi commentate fossero veramente nate come allegorie e che la lettera (amore per una donna) fosse una finzione che doveva servire a rendere il vero significato (amore per la filosofia) credibile, e scartare così l'uso della personificazione (Questioni 119). Ma non c'è niente nelle canzoni che possa suggerire siffatta interpretazione, e perciò, se si suppone che questa fosse stata l'intenzione originaria dell'autore, bisognerebbe concludere che Dante non credesse possibile manifestare la vera intenzione ("tutta tua vision fa manifesta", Par. 17.128) senza il sussidio di 
un commento in prosa da parte dello stesso autore. Nella Commedia Dante, insieme all'uso delle personificazioni e delle astrazioni, scarta anche quello del prosymetrum, lasciando alla poesia stessa il compito di esaminarsi e difendersi. In effetti, nella prassi poetica della Commedia si va delineando l'evoluzione del concetto del senso letterale, intuito ma malamente espresso nel Convivio, nella crescente consapevolezza che si tratta appunto di "senso", cioè già interpretazione in quanto struttura di significato del poema, costruzione intenzionale dell'autore, avvicinandosi, come avverte Pépin, alla definizione tomista (Summa Theologiae 1.1.10) che fa equivalere il senso letterale di un testo all'intenzione dell'autore, al significato della finzione poetica. ${ }^{9} \mathrm{E}$ d'altra parte il vero significato della storia biblica è estratto tramite lo stesso processo di interpretazione, poiché la lettera (la storia) è espressione dell'intenzionalità del suo Autore. Il dibattito tra proponenti dell'allegoria dei poeti e allegoria dei teologi ${ }^{10}$ assume che Dante abbia operato una scelta unilaterale tra l'Eneide e la Bibbia. Tale scelta è invece impraticabile se l'allegoria dei teologi ha come base una serie di fatti completamente veri, cosa che non si pud dire della Commedia, perché, accanto alla storia assunta a soggetto, c'è il racconto di un'esperienza i cui "fatti", a prescindere dalla realtà dell'esperienza della visione mistica di cui non c'è ragione di dubitare, non possono essere presi alla lettera, come pensarono bene di precisare vari commentatori trecenteschi, il Lana, l'Ottimo, Guido da Pisa, ${ }^{11}$ avvertendo il lettore che non si deve intendere che le persone che Dante dice di incontrare siano veramente nell'Inferno o altrove, perché a un essere umano non è dato di sapere queste cose. Pietro Alighieri usa termini più forti:

Nam quis sani intellectus crederet ipsum ita descendisse, et talia vidisse, nisi cum distinctione dictorum modarum loquendi ad figuram? Nam non est ipse literalis sensus ipse figura, sed id quod est figuratum. (Commentarium)

[Chi infatti, essendo sano d'intelletto, crederebbe che egli stesso fosse a tal modo disceso e avesse tali cose visto, se non distinguendo i modi del parlare figurato? Perché il senso letterale non è esso stesso una figura, ma quello che è figurato]. ${ }^{12}$

La figura è il significante, il senso letterale è il significato che essa esprime, e del resto Dante stesso ci dice che si tratta di "esempi" (Par. 17.139-42).

È inoltre impraticabile certamente a livello di stile perché la Bibbia può ispirare le finalita didattiche della poesia, espresse nell'ideale di lingua "piana", da cui Dante deriva la funzionalità della lingua poetica, non la stessa lingua o lo stile. Lo stesso Agostino (Confessioni 3.5.9) aveva dovuto ammettere la delusione che aveva provato nei confronti dello stile biblico, prima di capire che quello stile umile rivelava un contenuto sublime. Al di là della Bibbia, la produzione letteraria cristiana non pud, in generale, sia per contenuti che per stile, offrire a Dante un concreto modello letterario. La BattagliaRicci sostiene che spesso Dante sia arrivato al suo realismo attraverso il ser- 
mo humilis della Sacra Scrittura, che permetteva di introdurre la realtà quotidiana in un contesto concettuale elevato, e ne apporta alcuni esempi (206-19). $\mathrm{Ma}$, come gli esempi apportati dimostrano, si tratta di prestiti a livello di costruzioni metaforiche.

Dalla Bibbia egli ha appreso la funzionalita delle "mescolanze" lessicali, del plurilinguismo, del realismo appunto, ma non certo le costruzioni sintattiche e ritmiche, non lo stile poetico, per il quale egli si rivolge evidentemente ai classici.

Ma tomiamo al problema dell'allegoria. La critica ha spesso collocato al centro del dibattito la questione della autenticità dell'Epistola a Cangrande. ${ }^{13}$ Non ho in questo luogo alcuna intenzione di parteggiare a favore dell'una o dell'altra tesi perché, nonostante gli argomenti, spesso validi, dei critici, non mi sembra tuttora che ci siano prove sufficienti a convincermi in un modo o nell'altro. Diro solo che l'argomento basato sulle contraddizioni tra l'Epistola e il Convivio non puo provare assolutamente nulla, dato che sono state scritte in tempi diversi, prima e dopo la Commedia; anzi semmai a sfavore dell'autenticità, ma a livello di impressione e non di prova, è per me il fatto che in realtà essa cambia troppo poco, perché, se fu scritta a scrittura ultimata o inoltrata del Paradiso, direi che quelle formulazioni deludono alquanto dopo l'audacia di un poema come la Commedia.

Del Convivio essa mantiene la stessa teoria dei quaturo sensi, e gli stessi esempi, quello di Orfeo, e quello dell'Esodo, salvo che non si pronuncia più sulla differenza tra poeti e teologi, e, se è lecito dedurre qualcosa dal silenzio, forse vi si può vedere implicito il superamento della distinzione, divenuta ormai, come credo, irrilevante. Quello che cambia è lo sdoppiarsi del "soggetto", e a questo proposito ci si potrebbe chiedere, se l'Epistola non è di Dante, a quale commentatore e per quale ragione sarebbe venuta in mente una siffatta divisione.

Questa divisione è d'altronde meno semplicistica di quanto non appaia. I due soggetti rappresentano due ottiche diverse, la terrena, temporale, e la celeste, eterna, con cui si presenta il duplice ideale di città terrena e città eterna; la prima appena una possibilita che la cupidigia non permette di attuare, la seconda una realtà concreta e assoluta che l'uomo può meritarsi. Allo stesso tempo mentre l'esplorazione della prima si avvale della storia come lettera, la esplorazione della seconda, pur essendo realta oggettiva, deve avvalersi come lettera di un racconto inventato dal poeta. Lo sdoppiamento del soggetto, chiunque sia l'autore dell'Epistola, bene si attaglia all'opera cui veramente "han posto mano e cielo e terra", nella quale la realta fattuale e l'invenzione poetica si intrecciano collaborando alle stesse finalità.

Anche qui bisogna porre diversamente i termini della questione, spostandola nell'area specifica della intenzionalita. Quello che rende la Bibbia un'eccezione come scrittura, non è tanto che la lettera è di per sé vera, se anche l'Eneide è accolta come historia, quanto il fatto che la narrazione dei 
fatti rivela l'intenzione del suo Autore, e i suoi attori sono coscienti del senso della storia in cui agiscono; in essi c'è la consapevolezza d'esser parte di un disegno divino, che lo svolgersi degli eventi ha la sua finalità nella venuta del Messia. Il racconto del viaggio di Enea, storicamente vero, o così creduto, e presentato come partecipazione consapevole a un disegno che lo rende necessario, è in realtà, come racconto, un viaggio a ritroso nella storia già compiuta a cui sfugge il vero senso della sua provvidenzialità.

Il racconto della Commedia si svolge in entrambe le direzioni: quella della memoria e quella della speranza, per cui l'opera sottintende la tensione, non l'antagonismo, tra i due testi per eccellenza, la Bibbia e l'Eneide,${ }^{14} \mathrm{e}$ in questo processo di scrittura che riflette su se stessa, sulla propria lingua e finalità, si crea un terzo genere che accorcia la distanza tra i due testi. Il problema del controllo del significato del testo è risolto proprio tramite l'autoriflessione sul soggetto, sul metodo e sulle finalità del racconto stesso, perché essa conferisce a Dante poeta e personaggio una consapevolezza e intenzionalità pari a quelle degli agenti della storia e della scrittura del Vecchio Testamento, in quanto fede e preparazione alla seconda venuta del Messia. Non che Dante abbia d'altro canto rinunciato all'ideale monarchico, alla possibilità di felicità $\mathrm{e}$ giustizia terrena; lo spirito profetico che pervade tutta la Commedia è teso a questa seconda venuta di Cristo, e l'ideale civile rimane valido e necessario come parte della preparazione al regno divino. Lo spirito profetico non potrebbe d'altronde giustificarsi altrimenti, se non si vuole cadere nell'assurdo di prendere troppo alla lettera il contenuto, che Dante invece lascia vago, delle singole profezie e dover concludere che Dante è profeta ma le sue profezie non si avverano (Nardi, Dante 325). Dante non è profeta di singoli avvenimenti ma dell'Avvento in cui crede fermamente come gli ebrei credevano nella venuta del Messia. Dante, nelle vesti di profeta, di teologo, di mistico, non smette mai di essere poeta; la poesia acquista il valore di azione dettata dalla caritas nell'assumersi la missione della rivelazione del vero, nella ricerca del quale la ragione e la fede, la filosofia e la teologia si complementano. Quanto all'intenzionalità, il rapporto tra l'Eneide e la Bibbia è il rapporto tra "autore", autorità letteraria e sapienza laica, e "Autore" (Dio), autorità assoluta che garantisce la verità della visione profetica e che permette a Dante di costruire la sua opera con pieno controllo autoriale del suo significato.

Sul problema del senso letterale della Commedia le opinioni dei critici variano dalla adesione completa: "Dante credette che gli fossero mostrati in visione l'Inferno, il Purgatorio, il Paradiso terrestre, come veramente sono nella realta" (Nardi, Dante 308), alla più moderata formula del Singleton: la fictio della Commedia è quella di non essere una fictio (Singleton, "The Irreducible" 129; Hollander, Studies 84), alla proposta della Barolini di esaminare le strategie che Dante usa per farci credere vera la sua fictio (46). Parallelamente si hanno varie definizioni di fictio: "finzione allegorica" (Paolazzi 
62), "invenzione" (Marigo), "composizione" (Paparelli 134), in misura proporzionata all'importanza che si attribuisce alla teologia o alla poesia di Dante. Hollander infatti insiste che Dante è "theologus-poeta" e non "poetatheologus" (Studies 83), e Tateo afferma:

quando Dante si poneva il problema di come la scienza, quella divina soprattutto, potesse rivivere nella parola, o in un prodotto dell'uomo, e divenire comunicabile, non affrontava un problema di natura estetica; il problema era di natura squisitamente teologico-morale. (Questioni 140)

Il primo commentatore a prendere una posizione simile e Filippo Villani il quale scarta subito il poetico, ammettendo di non esservi molto versato: "Sed horum vestigationem peritis gramatice derelinquo" [ma lascio questa investigazione agli esperti di grammatica] e afferma che Dante non intendeva "docere gramaticam, sed moralem tradere philosophyam" [insegnare grammatica, ma trasmettere filosofia morale] (26).

Hollander del resto si riconosce pienamente in Villani: "I am aware that my championing of Filippo Villani is self-serving. Perhaps if dantisti had been reading him all these years this book would have been written long ago by someone else" (Allegory 296). Mi domando che cosa conferisca questa superiore auto-rità al testo del Villani; se i "dantisti" avessero letto Villani (Hollander sembra credere d'essere l'unico ad averlo letto), avrebbero (come hanno fatto) letto anche commentatori come il della Lana, l'Ottimo e il da Pisa per i quali invece la prima delle tre intenzioni dell'autore era proprio quella di "manifestare / dimo-strare / insegnare (discant)", "polida parlatura / ornato parlare di rettorica e ritmico / polite et ordinate loqui" (JenaroMacLennan 62-63). Il Villani è anche il primo a insistere sull'allegoria dei teologi, mentre gli altri si limitavano a spiegare etimologicamente il significato del termine. ${ }^{15}$ A questo punto si vede che il conflitto non è tanto tra diversi tipi di allegoria quanto tra teologia e poesia, come se per valorizzare l'una fosse necessario sopprimere l'altra. Niente potrebbe essere più lontano dalle intenzioni dantesche: se egli fosse stato solo mistico, ci avrebbe presentato solo la visione beatifica, e se fosse stato solo teologo, avrebbe scritto un trattato in prosa che forse pochissimi leggerebbero tutt'oggi. Invece se le sue parole e immagini continuano a convincerci al punto da farci credere che egli sia veramente stato nell'Inferno, nel Purgatorio, nel Paradiso, è grazie alla poesia e nient'altro. Cosa che Boccaccio aveva capito benissimo:

Adunque, acciò che con fatica acquistata fosse più grata, e perciò meglio si conservasse, li poeti sotto cose molto ad essa contrarie apparenti la nascosero; e perciò favole fecero, più che altra coperta, perché la bellezza di quelle attraesse coloro li quali né le dimostrazioni filosofiche, né le persuasioni avevano potuto a sé tirare. (Trattalello 620) 
Sono d'accordo col Paolazzi (52 sgg.) nel pensare che siano stati i critici a semplificare e irrigidire l'opposizione tra Bibbia e letteratura classica, tra teologia e poesia. Il problema è d'altronde di vecchia data, come sentiamo di nuovo nella difesa di Boccaccio:

Dico che la teologia e la poesia quasi una cosa si possono dire, dove uno medesimo sia il suggetto; anzi dico di più: che la teologia niuna altra cosa è che una poesia di Dio. $E$ che altra cosa che poetica finzione, nella Scrittura, dire Cristo essere ora un leone e ora agnello e ora vermine, e quando drago e quando pietra, e in altre maniere molte, le quali volere tutte raccontare sarebbe lunghissimo? che altro suonano le parole del Salvatore nello evangelio, se non uno sermone dai sensi alieno? il quale parlare noi con più usato vocabolo chiamiamo 'allegoria'. Dunque bene appare, non solamente la poesì essere teologia, ma ancora la teologia essere poesia. (Trattatello 621)

"Dove medesimo sia il suggetto" - è il caso della Commedia, la quale, forse così chiamata per pudore di chiamarsi "teodia", è la prima vera epopea cristiana, e forse l'unica opera in cui tutti i livelli di realtà umana e tutti i mezzi di conoscenza confluiscono nella materia della poesia in un atto di carità e di speranza.

\section{2) Tragedia e commedia}

Se "teodia" è il nome segreto dell'opera, il titolo effettivo va giustificato. Non sembra che i commentatori trecenteschi fossero sorpresi, come lo sono io, dalla straordinarietà del titolo, eppure non mi risulta che fosse pratica comune denominare la propria opera col nome di un genere senza qualificarlo. Essi comunque sentono di doverlo spiegare, e lo fanno solitamente in termini di stile e di trama, e allo stesso modo sentono di dover spiegare anche perché l'Eneide è una "tragedia": o perché termina con la morte di Turno, o perché parla della distruzione di Troia (non la fondazione dell'impero romano); il che farebbe pensare che né quella attribuzione, né i concetti di "tragedia" e "commedia" fossero cosa pacifica. ${ }^{16}$ Il più tormentato di tutti su questo punto e Boccaccio. Combattuto tra la propria convinzione che il titolo dell'opera non si addice allo stile, e la necessità che sente di doversi piegare (ma malvolentieri) ad ammettere la superiorità del latino, ${ }^{17} \mathrm{e}$ allo stesso tempo di difendere Dante dall'accusa di aver scritto in volgare perché non sarebbe stato capace di farlo in latino, finisce anch'egli coll'optare per la convenienza del titolo rispetto alla trama. Mi sembra di intravvedere nelle sue parole perfino il dubbio che Dante avesse veramente dato quel titolo:

E così, acciò che fine pognamo agli argomenti, pare, come di sopra è detto, non convenirsi a questo libro nome di 'comedìa'. Né si può dire non essere stato della mente dell'autore che questo libro non si chiamasse 'comedì', come talvolta ad 
alcuno d'alcuna sua opera è avvenuto, con ciò sia cosa che esso medesimo nel XXI canto di questa prima cantica il chiami Comedia. (Esposizioni 6)

I critici moderni, anche a prescindere dalla loro opinione sull'autenticità dell'Epistola, tendono generalmente a dare poco peso alle considerazioni relative alla trama, e bisogna ammettere che questo motivo, anche se Dante lo considerasse parte del significato di commedia e tragedia, non soddisfa perché non spiega granché del contenuto, significato o finalità dell'opera, ovvero spiega solo l'ovvio. Inoltre, se la trama comica (triste inizio e lieto fine) si adegua a quella della Commedia, quella tragica (lieto inizio e triste fine) non sembra applicabile all'Eneide. E questa è l'opinione della maggior parte dei critici, ${ }^{18}$ con l'eccezione di Hollander, secondo il quale "La Commedia è una commedia; per Dante l'Eneide è una tragedia", perché riflette il tragico fallimento di Virgilio: "La grande fons, l'Eneide, testo salvifico per Stazio, rimane libro chiuso per il suo stesso autore. In un contesto cristiano nessun fallimento letterario potrebbe essere più tragico" (Il Virgilio 70).

Nel De Vulgari $(2.4 .5-8 ; 12.3 .6,8)$ Dante basa la distinzione unicamente sul registro stilistico: alto quello della tragedia e medio o umile quello della commedia, e, rifacendosi all'autorità di Orazio, fa corrispondere ai tre generi, tragedia, commedia e elegia, il volgare illustre, medio e umile. Lo stile è relativo alla maggiore o minore gravità della sententia ed eccellenza dei versi, dei vocaboli e delle costruzioni; ma allo stesso tempo egli prevede l'opportunità di mescolanze, purché queste non intacchino l'integrità della lingua. L'esperienza poetica impedisce che la teoria si irrigidisca in regole esterne e irrevocabili, e Dante dà maggior peso alla prassi: l'imitazione dei "poeti regolati" e la "gentile discretio" dell'artista. ${ }^{19}$ Scegliere il titolo di "Commedia" è percio darsi piena libertà (Contini 104) d'esercizio della propria discretio nell'organizzazione della materia dell'opera, la cui eterogeneità giustifica, anzi rende necessario il plurilinguismo.

In che senso è però "medio" lo stile? Se teniamo conto del principio della convenientia (congruenza tra registro stilistico e la materia: situazioni, personaggi, concetti da rappresentare ed esprimere), è necessario concludere che lo stile della Commedia è anch'esso eterogeneo? Mentre le variazioni di tono e di lessico fungono da colorature stilistiche, l'eterogeneità vera e propria risiede a livello del linguaggio, del lessico, non dello stile che rimane unico, controllato dall'uso della terza rima e caratterizzato da costanti ritmiche e sintattiche tipicamente dantesche:

Per quel che riguarda la sintassi, si può ben dire che essa, a differenza del linguaggio, non discende mai di livello, non cessa mai di adempiere ad una sua funzione nobilmente ordinatrice [...] [Essa] si presenta come un organismo insieme solido e articolato, con sue distinte nervature, in cui la linea del discorso si dirama senza tuttavia venir mai meno alla sua mirabile unità di impianto [...] In questo senso Dante 
ha saputo accogliere gli insegnamenti della tradizione classica [...] e insieme della tradizione scolastica, così attenta alle strutture razionali del discorso. (Blasucci 679) ${ }^{20}$

Vale la pena notare che il plurilinguismo è legato di volta in volta alla situazione, alla realtà specifica da rappresentare, alla misura e al genere di reazione emotiva espressa dal personaggio o in cui è coinvolto il lettore, e non al personaggio (non ci scno cioè tanti linguaggi quanti sono i personaggi), ${ }^{21}$ né quindi al luogo. Vocaboli e suoni del registro umile, plebeo, non sono infatti appannaggio della prima cantica. ${ }^{22}$

Se diciamo che lo stile è "medio" perché in tensione tra l'alto (l'Eneide) e l'umile (la Bibbia), la conclusione non varia: nella prassi della Commedia gli schemi rigidi descritti dalla precettistica e ai quali Dante aveva inizialmente, ma non senza riserve, aderito, non reggono più. Il rapporto tra stile, lingua e materia dipende interamente dalle finalità del poeta, come la riuscita della poesia dipende interamente dalle capacità dello stesso, a prescindere da formulazioni e conoscenze teoriche. In altre parole, la possibilità di "mescolanze" e la necessità di dover talora contravvenire alle regole, che Dante aveva sempre previsto grazie alla esperienza viva di poeta (non già solo di critico), vengono corroborate dalla costatazione che, con buona pace dei manuali di retorica, le opere letterarie non corrispondono alle regole. Lo stile elevato della letteratura classica può essere usato per esprimere una realtà raccapricciante (le furie troiane e tebane), e lo stile umile biblico per esprimere concetti sublimi; lo stile medio della Commedia esprime quindi un ideale ancor più che una realtà (dello stesso stile). Chiamare l'Eneide tragedia con l'aggiunta, in apparenza superflua, dell'aggettivo "alta" (Inf. 20.113), vuol dire attirare l'attenzione sulla inadeguatezza dei limiti impliciti nella nozione di "genere". L'Eneide ha lo stile elevato solitamente attribuibile all'area del tragico, ma, quanto a genere narrativo, non può essere una tragedia. ${ }^{23}$

I manuali di retorica, da Cicerone al Garlandia, descrivono tre generi di narrazione, fabula, historia e argumentum:

De Inventione Fabula est, in qua nec verae nec verisimiles res continentur. Historia est gesta res, ab aetatis nostrae memoria remota. Argumentum est ficta res, quae tamen fieri potuit.

[La 'fabula' narra cose né vere né verosimili. La 'historia' narra fatti accaduti in epoca remota. Lo 'argumentum' narra cose inventate, che però potrebbero accadere.]

Rhetorica ad Herennium Fabula est, quae neque veras neque verisimiles continet res, ut eae sunt, quae tragediis traditae sunt. Historia est gesta res, sed ab aetatis nostrae memoria remota. Argumentum est ficta res, quae tamen fieri potuit, velut argumenta comoediarum.

[La 'fabula' narra cose né vere né verosimili, come quelle trattate nelle tragedie. La 'historia' narra fatti accaduti, ma in epoca remota. Lo 'argumentum' narra cose inventate, che però potrebbero accadere, quali gli argomenti delle commedie.] 
Poetria Fabula est que nec res veras nec verisimiles continet. Historia est res gesta ab etatis nostre memoria remota. Argumentum est res ficta que tamen fieri potuit, ut contingit in comediis.

[La 'fabula' narra cose né vere né verosimili. La 'historia' narra fatti accaduti in epoca remota. Lo 'argumentum' narra cose inventate, che perd potrebbero accadere, come avviene nelle commedie.] (Paolazzi 90-91)

Come vediamo, il genere narrativo della commedia è ficta res, che non solo è verosimile, ma potrebbe anche essere vera, mentre quello della tragedia è la fabula. È possibile che Dante consideri la res dell'Eneide né vera né verosimile? C'è poi una cosa che lascia perplessi nella definizione di historia: perché la gesta res appartiene a "memoria remota"? Dove situare allora un'opera che narra la gesta res di epoche e personaggi recenti (come avviene nella Commedia)? Se questa è la tradizione retorica ereditata da Dante, dovrebbe meravigliarci di più che un'opera riuscisse ad attenersi fedelmente agli schemi. Si può dire invece che per Dante, proprio nell'appellarsi alla tradizione, si presentasse la possibilità di mettere in gioco tutta la problematica relativa al rapporto tra storia e letteratura; può proporre alla pari l'opera virgiliana e la propria come historia ed esplorarne, tramite il confronto con la Sacra Scrittura, le differenze. La Commedia, assumendo come soggetto la storia recente, riprende e continua il racconto dell'Eneide; allo stesso tempo, accogliendone la metafora del viaggio, il soggetto del racconto diviene ficta res. Entrambe le opere sono dunque una mescolanza di generi narrativi, ma la mescolanza avviene in modo diverso in ciascuna: per la Commedia è un trapassare del soggetto dall'area dell'historia a quella dell'argumentum (e viceversa), i limiti tra i quali sono del resto già sfumati dall'ambiguità del fieri potuit; per l'Eneide il movimento sarà invece tra l'area dell'historia e quella della fabula. Quando anche il proprio parlare corre il pericolo di sembrare favoloso, Dante interviene, come nel XVI dell'Inferno (118-36), con un appello diretto al lettore intimandogli di credere come vero l'inverosimile: “... e per le note / di questa comedìa, lettor, ti giuro ..." (12728).

Insomma, nel chiamare l'opera virgiliana "tragedia" e la propria "commedia", Dante paradossalmente le avvicina e le allontana contemporaneamente, e la differenza e, inaspettatamente, rivendicata nell'ambito della invenzione poetica: l'una, come il mito stesso, è una specie di "non falso errore", l'altra una verità che ha "faccia di menzogna", distinguendo così la finzione retorica in ordine alle finalità didattiche, asservita e compartecipe all'assoluta verità divina.

Il riconoscimento di queste mescolanze è evidente nel commento di Pietro Alighieri, lettore attento e colto, il quale distingue sette sensi della scrittura invece dei soliti quattro: superficiale o parabolico, storico, apologetico, metaforico, allegorico, tropologico, anagogico. I punti interessanti sono: 1) inclu- 
de nello storico non solo res veras, ma anche verisimiles; 2) la res dell'apologetico non è né vera né verosimile, ma inventata e ha funzione transumptiva "secundum aliquam similitudinem" [secondo qualche similitudine]; essa si distingue però da quella della fabula, "quae nihil informationis habet nisi vocem" [che non ha altro contenuto che le parole]; 3) include il metaforico, distinguendolo dall'allegorico (Commentarium 4-8).

Si può vedere qui un approccio più critico alla nozione di historia, ormai spoglia della condizione "ab aetatis nostrae memoria remota", e assimilata al modo dell'argumentum che lasciava un ampio margine di scelta tra il vero e il verosimile ("quae fieri potuit"). Inoltre l'inclusione del metaforico e il rilievo dato alla similitudine, attestano una sensibilità poetica più spiccata e più informata: la Commedia non è la Bibbia ma opera poetica che dei modi poetici deve usufruire.

La Carugati parla di parrhesia, cioè la possibilita che ha il credente di 'rifare' la Sacra Scrittura, o per lo meno di affiancarle un testo, il proprio, costruito sulla verità tanto quanto la parola di Dio" (240), ma aggiunge che Dante

non tollera di essere semplicemente assimilato agli scrittori spirituali del medioevo [... ] per la coscienza linguistico-retorica che lo sorregge. Non a caso Dante nasce alla scrittura prima di approdare alla mistica [...]. Abbiamo visto anche che il 'mistico puro' è consapevole, intrinsecamente, costitutivamente, della artificiosità della sua scrittura. Ma forse è più del mistico-poeta disposto a 'terminare' [...]. Il mistico-poeta ( $m a$ forse in questa categoria non c'è che Dante a rientrare) oscilla tra il silenzio e un linguaggio che, per il fatto di essere così coscientemente il prodotto di una ricerca, di un'arte, rischia di scollarsi dalle ragioni ultime, vivendo per così dire da solo, di una vita tragicamente precaria. [...] Quello (il libro) di Dante [...] è riuscito per tre cantiche a superare ogni 'passo forte' e sta quasi blasfemo, al di là del vero e del falso, a trascinarci nella propria fragilità. $(247-48)^{24}$

\section{Il Barański invece esclude che Dante pensasse al suo poema come a una nuova Bibbia:}

Il suo fu piuttosto un atto di debita e rispettosa imitatio nei riguardi di ciò che per lui era il testo supremo. Seguendo l'esempio della Bibbia, Dante riuscì non solo ad indicare a tutti gli uomini la via della salvezza in conformità alla volontà divina, ma anche a sfuggire egli stesso, come poeta, alle insidie delle convenientiae retoriche. $\mathrm{Ma}$ Dante volle anche far vedere che egli, come uomo, non poteva raggiungere quella sintesi perfetta di verba, res e signa che contraddistingue la scrittura divina. Perciò, egli non si limitò ad imitare solo la forma biblica; ma, nel tentativo di adeguare la debole parola umana alle verità divine di cui era stato fatto portatore, e per sottolineare il carattere umano della sua poesia, egli assimilò qualsiasi testo scritto "per trattar del ben" nel modo più efficace; così, assieme alla allegoria biblica, come abbiamo visto, egli unì altre tradizioni allegoriche, per creare, anche per l'allegoria, uno stile 'comico'. (95-96) 
L'originalità della Commedia consiste nello strutturare poeticamente l'esplorazione di diversi generi di esperienza e di diversi livelli di realtà, nell'attraversare e farsi attraversare da diversi testi e, per questo, nel non assomigliare a nessuno. Nella categoria del mistico-poeta (o del teologo-poeta, se lo si preferisce) non c'è che Dante, ed è per questo che è così difficile cercare di descrivere l'opera piegandola e adeguandola a distinzioni e definizioni troppo limitate e contraddittorie. In essa l'estetico e il teologico sono inseparabili sia a livello della visione che a quello dell'espressione che è (e sembra assurdo doverlo ricordare) "poetica".

Dire che l'ispirazione è divina e identificare l'Amore con lo Spirito Santo, non comporta l'esclusione o il far passare in secondo ordine l'esperienza estetica e poetica; nel Paradiso Dante sceglie Davide, il "cantore dello Spirito Santo", proprio in qualità di "cantore". La "teodia" è canto che celebra la bellezza dell' arte divina ("E pero dice lo Salmista: 'Li cieli narrano la gloria di Dio, e l'opere sue annunzia lo fermamento"'; Conv. 2.5.13) nella cui contemplazione non si distinguono l'esperienza mistica e quella estetica poiché nell'arte divina non c'è distinzione tra bellezza e bontà. I principi e le finalità della poetica dantesca si ispirano perciò direttamente all'arte divina e l'organizzazione poetica si basa sulla imitazione di essa, per la similitudine che si stabilisce tra la struttura di significati del libro della memoria e quella del volume divino che per l'universo si squaderna e in cui Dio si manifesta.

I luoghi danteschi che riflettono questi parallelismi tra i modi di conoscenza (intelligendi) e i modi di espressione (significandi), e tra l'arte divina e l'arte umana sono numerosi:

1) la natura è arte divina (Mon. 1.3.2; Inf. 11.99-100), e l'arte umana, che imita la natura, è quasi nipote di Dio (Inf. 11.103-05);

2) sia l'arte umana che quella divina hanno tre gradi: nella mente dell'artefice, nello strumento, e nella materia; la natura è prima nella mente di Dio, poi nel cielo come strumento mediante il quale la materia viene informata a similitudine della bontà divina (Mon. 2.2.2);

3) grazie a questa similitudine, si può indirettamente conoscere la bontà e la volontà divina, poiché l'universo è "vestigium" (Mon. 1.8.2), cera su cui è impresso un occulto "sigillo" (Mon. 2.2.8); per cui si pud dedurre, abbinando le premesse 1) e 2), che la struttura di un'opera d'arte rivela l'idea, l'intenzione che era nella mente dell'artefice;

4) cio che rende l'universo somigliante a Dio è la sua forma costituita dalle correlazioni tra le cose (ordine):

[...] Le cose tutte quante hanno ordine tra loro, e questo è forma che l'universo a Dio fa simigliante

(Par. 1.103-05. Cfr. 29.31-36) 
la quale è conoscibile perché "l'intelletto umano potenzialmente a sé adduce tutte le forme universali" (Conv. 4.21.5). ${ }^{25}$ L'ordine gerarchizzato di tutte le cose fra di loro implica un'interdipendenza che valorizza il carattere e le finalità di ciascuna, relative alla specie cui appartiene e alla specificità individuale, ${ }^{26} \mathrm{e}$ non comporta perciò fissità e determinismo ma provvidenzialità che ha come fine il bene, la felicità, ${ }^{27}$ per cui l'adesione a questo ordine provvidenziale non è un abdicare alla propria libertà ma conquistarla in quanto è l'unico modo di essere se stessi, di esprimere a pieno la propria natura:

tal mi sembiò l'imago de la 'mprenta

de l'etterno piacere, al cui disio

ciascuna cosa qual ell'è diventa.

$(\text { Par. 20.76-78) })^{28}$

Ogni cosa infine non è che lo "splendor di quella idea / che partorisce, amando, il nostro sire" (Par. 13.53-54); ogni cosa dunque, riflettendo la luce divina, riporta all'uno donde proviene. Similmente nel sistema di correlazioni che l'opera poetica stabilisce tra i suoi elementi (immagini, motivi, suoni, ecc), ciascuno di essi ha funzione strutturale; ciod ogni parte riporta al tutto, riflettendo l'idea globale dell'autore.

5) L'intelletto umano, che è la parte più nobile dell'anima perché partecipe della divinità, ${ }^{29} \mathrm{e}$ il cui bene è la conoscenza della verità, ${ }^{30}$ trova nella contemplazione della forma dell'universo la sua beatitudine:

La forma universal di questo nodo credo ch'i' vidi, perché più di largo, dicendo questo, mi sento ch' $i$ ' godo.

(Par. 33.91-93)

\section{Il desiderio del bene e il desiderio di Dio si identificano:}

E però che naturalissimo è in Dio volere essere - però che, sì come ne lo allegato libro si legge, 'prima cosa è l'essere, e anzi a quello nulla è' - , l'anima umana essere vuole naturalmente con tutto desiderio; e però che 'l suo essere dipende da Dio e per quello si conserva, naturalmente disia e vuole essere a Dio unita per lo suo essere fortificare. E però che ne le bontadi de la natura e de la ragione si mostra la divina, viene naturalmente l'anima umana con quelle per via spirituale si unisce, tanto più tosto e più forte quanto quelle più appaiono perfette: lo quale apparimento è fatto secondo che la conoscenza de l'anima è chiara o impedita. E questo unire è quello che noi dicemo amore, per lo quale si può conoscere quale è dentro l'anima, veggendo di fuori quello che ama. (Conv. 3.2.7-9)

6) Il profondo legame tra la conoscenza "l'atto che vede" e l'amore "quel che ama" (Par. 28.109-11) si fonda sulla percezione del bello: le bellezze del creato sono un "logoro" col quale Dio attira a sé l'uomo ${ }^{31}$, per cui il bello è, 
nell'arte umana come in quella divina, mezzo di attivazione del desiderio e di persuasione.

Quella cosa che più adorna e commenda l'umana operazione, e che più dirittamente a buon fine la mena, sì è l'abito di quelle disposizioni che sono ordinate a lo inteso fine. [...] Quella cosa dice l'uomo essere bella, cui le parti debitamente si rispondono, per che de la loro armonia resulta piacimento. Onde pare l'uomo essere bello, quando le membra debitamente si rispondono; e dicemo bello lo canto, quando le voci di quello, secondo debito de l'arte, sono intra sé rispondenti. Dunque quello sermone è più bello, ne lo quale più debitamente si rispondono le parole. (Conv. 1.5.4,13-14) ${ }^{32}$

Il piacere estetico deriva dalla percezione dell'armonia la quale a sua volta rivela il fine a cui è ordinata: il bello è forma del bene..$^{33}$

In conclusione, l'ispirazione per Dante non è che questa lieta percezione dell'arte divina, dalla quale l'arte umana ricava e ne imita i principi di armonia responsabili sia della struttura dell'opera poetica che delle sue finalità: persuadere l'uomo a sollevare lo sguardo al cielo, farlo innamorare delle sue bellezze, riattivare il desiderio di bene, offrirgli coll'esempio della divina armonia il modello di giustizia e felicità terrena. Su questa similitudine tra arte umana e arte divina si fonda il realismo dantesco, inteso come esempio concreto che adombra l'ineffabile.

\section{La Trobe University}

\section{NOTE}

1 Per A. Cassell significa solo trovarsi di nuovo, cioè ricadere, nel peccato (14). Questa interpretazione non contraddice affatto la mia, poiché non vedo perché sia necessario escludere il significato di tomare alla coscienza: se Dante ci dice che è ricaduto nel peccato, è ovvia una presa di coscienza, la quale, del resto non vuol dire liberarsi automaticamente del male senza bisogno della grazia e di intraprendere il viaggio, come crede Cassell (11); il desiderio di liberarsene è prerequisito del viaggio. Sono d'accordo con Cassell che è necessario distinguere tra Dante personaggio e Dante poeta (10), ma se il Dante personaggio può essere ricaduto nel peccato, il Dante poeta si trova a dover trovare un altro modo di 'dire'; il viaggio di conoscenza appartiene cioè a entrambi. C'è infine una differenza fondamentale tra sonno e sogno e visione: questi ultimi due sono rivelazione parziale, intuitiva, di una verità di cui ci si può ricordare e che si può narrare, mentre il sonno è negazione della memoria e rende il viaggio impossibile.

2 "Così l'adolescente che entra ne la selva erronea di questa vita, non saprebbe tenere lo buono camino, se da li suoi maggiori non li fosse mostrato" (Conv. 4.24.12).

3 "Senza conoscere" nel senso di essere nati prima della sua venuta, perché per salvarsi è necessario "credere" in Cristo: "A questo regno / non salì mai chi non credette 'n Cristo, / vel pria vel poi ch'el si chiavasse al legno" (Par. 19.103-5), ma è possibile credere in lui anche "pria" che si sacrificasse per l'umanità.

4 Collocando Marzia nel Limbo, Dante modifica l'interpretazione che di lei dà nel Convivio (4.28.13-19) quale l'anima nobile che toma a Dio. Non è facile giudicare il contenuto esatto e la portata di questo cambiamento, ma è probabile che questa storia abbia subito lo stesso trattamento della profezia virgiliana. La verità della interpretazione allegorica della vicenda storica non dipende dalla intenzionalità dei suoi personaggi: la Marzia storica è solo tomata a Catone non a 
Dio, e Catone non è certo Dio e solo per grazia straordinaria si salva.

5 Consoli e Ronconi; Nardi, Dante 283; Hollander, Studies 72. Questa opinione è sorretta dalle parole di Dante nella Monarchia 2.3.

6 Si vedano: Conv. 3.15.7-9; Par. 24.64-81.

7 Sulle varie interpretazioni della figura di Virgilio, oltre al già citato volume di Comparetti, si consultino: Hollander, Allegory, in particolare 96-103, e Il Virgilio; Cassell 77-99; Consoli e Ronconi.

8 Hollander, Allegory 33. Cfr. D'Andrea, in particolare la nota 18, dove, rispondendo a Hollander, afferma "Non è la combinazione dei due tipi di allegoria a fare della poesia, nel Convivio, qualcosa di ibrido [...], ma la stessa esperienza letteraria nella sua complessità, imperfettamentre riflessa nello specchio della tradizione retorica, a suggerire la ricognizione di una diversa tradizione esegetica e la combinazione dell'una e dell'altra" (78). Cfr. anche Grayson (153). Si veda inoltre Simonelli.

9 Cfr. Pagliaro: "non è criticamente possibile separare nella Commedia la lettera e l'allegoria" (694).

10 Tra i sostenitori dell'allegoria dei teologi si segnalano: Singleton, Commedia; Nardi, Il punto; Mineo; Hollander, Allegory e Studies; Tateo, Questioni; Mastrobuono; Grayson; Cassell. Spesso coloro che si occupano della poesia non si pongono apertamente il problema della scelta, oppure minimizzano la divergenza tra le due, quali Mazzotta; Paolazzi. Cfr.: Greene; e D’Andrea.

11 Jenaro-MacLennan (63-64). Sullo sviluppo della teoria e della critica letterarie nel Medioevo, si consultino: Minnis and Scott; e Minnis.

12 Tutte le traduzioni dal latino sono mie.

13 Tra i sostenitori dell'autenticità si consultino: Moore; Barbi; Mazzoni; Hollander, Il Virgilio e Dante's Epistle; Paolazzi, in particolare 3-81. Tra coloro che ne negano invece l'autenticità: D'Ovidio; Pietrobono; Nardi,Il punto; Dronke, in particolare 103-11; Kelly. Infine c'è chi non si pronuncia, come Jenaro-MacLennan, e chi ritiene la questione irrilevante, come Eco, "L'Epistola XIII".

14 Non vi vede antagonismo neppure Aversano, che pure basa rigidamente tutte le sue tesi e dimostrazioni sulla "règle des oppositions".

15 Per quanto riguarda la nozione di allegoria elaborata dai trattatisti, si veda Alessio.

16 Né era del resto facile applicare le formule tradizionali alle opere in volgare: "La teoria retorica dei tre stili, umile, medio e sublime, consacrata dalla 'ructa di Virgilio', è di applicazione difficile alla lingua volgare. Si costatano senza dubbio, tra certi 'esti o gruppi di testi, differenze di tono, definibili a livello della sintassi, del lessico o delle figure. Ma questi cont rasti sono poco marcati, molto instabili, e non sarebbe possibile fondare su di essi un'opposizione pertinente. Qualificare di sublime, per l'abbondanza di tropi, lo stile della canzone di troviero o dei romanzi di Chrétien de Troyes, di mediocre quello di Maria di Francia e di umile quello di moltifabliaux non sarebbe che uno scherzo di cattivo gusto. La lingua romanza si piega male a queste distinzioni prese dalla tradizione antica" (Zumthor, Semiologia 159).

17 Credo sia da escludere che Dante attribuisse questo titolo alla sua opera perché scritta in volgare, dopo anni di propaganda in favore del volgare, e dopo la composizione del poema col quale sperava di meritarsi l'alloro. D'altra parte neppure questa considerazione prova definitivamente la non-autenticità dell'Epistola, in quanto, malgrado le sue convinzioni, Dante non può sottrarsi all'accettazione generale della superiorità del latino, sostenuta a lungo dopo la sua morte (basti vedere l'ostinazione di un Petranca e il dilemma di Boccaccio). Nello stesso Convivio, dove cerca di dimostrare che per il volgare si possa manifestare "altissimi e novissimi concetti convenevolmente, sufficientemente e acconciamente", sente di dover aggiungere "quasi come per esso latino" (1.10.12); e nelle Ecloghe deve nuovamente difendere le "comica verba" derise da Mopso (Giovanni del Virgilio), al quale conta di far cambiare idea con la presentazione della terza cantica, pecora gratissima che non appartiene a nessun ovile (= una fuori-genere per eccellenza, anche rispetto all'intero poema). L'Epistola insomma, chiunque sia l'autore, dimostra, con disperazione del Boccaccio, che il dibattito era vivo e a difendere la poesia in volgare non potevano essere le parole ma i fatti. E sappiamo che anche $\mathrm{i}$ fatti (la grande poesia della Commedia) non sempre ruscirono a convincere tutti; si veda il Petrarca, e si vedano le varie 
"difese" che punteggiano la fortuna del poema (si consulti a proposito Petrocchi).

18 Si vedano Mengaldo; e Tateo, "La teoria".

19 "Si può ben dire che, diversamente dalle poetiche mediolatine, al centro del discorso dantesco non è l'astratta eloquentia, sono i concreti eloquentes. Di qui un 'interiorizzazione delle norme della poetica, in cui è da vedere una delle ragioni più consistenti della novità del trattato. La validità delle norme non riposa più, alla fine, su criteri oggettivi e prefabbricati, ma sulla dignità dell'esperienza poetante che le fonda con la propria autorità" (Mengaldo 58).

20 Similmente Risset (63).

21 La Battaglia-Ricci (40) concorda con la tesi di Contini che confutava quella di Spitzer, secondo cui la "retorica" aveva come funzione la caratterizzazione del personaggio. Anch' io credo che Dante "presti" la sua retorica ai personaggi e che quindi non si tratti di linguaggi distinti, ma mi sembra che si possa dire ugualmente che essa riesca a caratterizzarli nel senso che riflette una certa abitudine mentale e senz'altro il modo in cui essi intendono presentare la propria situazione. Così, per esempio, la tecnica dell'amplificazione e quella dell'abbreviazione riflettono compiutamente mentalità e intenzioni di Buonconte e di Pia rispettivamente (Purg. 5.85-136). Al pathos unito alla logica della propria fine e insieme allo sfoggio delle proprie conoscenze geograficoatmosferiche del primo, la seconda contrappone implicitamente un mondo più piccolo, privato, entro le due abitazioni prima e dopo il matrimonio, e alla sua reticenza sono affidati e l'espressione del chiuso pudore e della vergogna della propria vicenda, e il discreto sfoggio, rispetto al precedente, di una più raffinata, più profonda cortesia.

22 Si vedano per esempio i seguenti luoghi: Purg. 14.43-51 (Guido del Duca); Par. 17.124-32 (Cacciaguida); 27.22-27 (San Pietro); 29.103-29 (Beatrice).

23 Non lo è neanche per quanto riguarda la trama: la morte di Turno conferisce al finale una nota più patetica che tragica. Inoltre se si suppone che il senso del viaggio di Enea sia la fondazione di Roma, il contenuto tragico diventa omologabile alle "altre cose" della Commedia, il che farebbe diventare anche l'Eneide una "commedia". Ci si potrebbe chiedere se la celebrazione della gloria di Roma fosse la vera intenzione di Virgilio nello scrivere l'Eneide, ma è una domanda che sconfina dai limiti della presente discussione, perché non sembra che Dante se la sarebbe posta.

$24 \mathrm{Si}$ veda anche Corti, in particolare 16-20 dove parla del collegamento con l'universo e il linguaggio dei mistici.

25 Cfr. Purg. 25.62-66; Par. 33.91 sgg.

26 Conv. 2.4.4-5; Mon. 3.12; Par. 2.111-41; 6.124-26; 18. 111.

27 Mon. 2.2.2; Par. 8.97; 13.58.

28 Cfr. Purg. 16.64-81; 18.40-75; Par. 1.106-14; 5.19-30; 8.122-48; 17.37-42.

29 Conv. $2.4 .14 ; 3.2 .14 ; 4.15 .11 ;$ Par. $4.40-42 ; 19.52-54$.

30 Conv. 2.13.6; Par. 4.124 sgg; 28.106-14.

31 Purg. 19.61-69; cfr. 14.148-51.

32 Cfr. Conv. 3.15.11-14; 4.25.12-13.

33 Questo concetto è molto simile a quello tomista: "Nam bonum proprie respicit appetitum: est enim bonum quod omnia appetunt. Et ideo habet rationem finis: nam appetitus est quasi quidam motus ad rem. Pulchrum autem respicit vim cognoscitivam: pulchra enim dicuntur quae visa placent. Unde pulchrum in debita proportione consistit: quia sensus delectatur in rebus debite proportionatis, sicut in sibi similibus; nam et sensus ratio quaedam est et omnis virtus cognoscitiva. Et quia cognitio fit per assimilationem, similitudo autem respicit formam, pulchrum proprie pertinet ad rationem causae formalis" (Thomas Aquinas 1.5.4, ad 1). [II bene riguarda propriamente il desiderio: è infatti buono quello che tutti desiderano. E dunque ha l'aspetto di un fine: ché il desiderio è come un movimento verso la cosa. Il bello, d'altra parte, riguarda la facoltà conoscitiva: infatti si dicono belle le cose piacevoli a vedersi. Per cui il bello consiste nella debita proporzione: perché i sensi si dilettano delle cose debitamente proporzionate, in quanto simili a sé; poiché anche i sensi sono una specie di proporzione così come ogni facoltà conoscitiva. E poiché la conoscenza avviene per assimilazione, e la similitudine riguarda la forma, il bello propriamente appartiene alla natura della causa formale.] Si consultino a proposito: Zumthor, Langue, specialmente 221-22; Eco, Il problema e Arte e bellezza. 


\section{OPERE CITATE}

Alessio, Gian Carlo. "L'allegoria nei trattati di grammatica e di retorica". Picone 21-41.

Alighieri, Dante. Opere. Ed. Fredi Chiappelli. Milano: Mursia, 1978.

L La Commedia secondo l'antica vulgata. Ed. Giorgio Petrocchi. Torino: Einaudi, 1975.

Alighieri, Pietro. Commentarium Petri Allegheri super Dantis ipsius genitoris comoediarum. Ed. Vincenzo Nannucci. Firenze: Garinei, 1946.

Ascoli, Albert. "The Vowels of Authority (Dante's Convivio IV.vi.3-4)". Discourses in Authority in Medieval and Renaissance Literature. Ed. Kevin Brownlee and Walter Stephens. Hanover: UP of New England, 1989. 23-46.

Aversano, Mario. Il velo di Venere. Allegoria e teologia dell'inmaginario dantesco. Napoli: Federico e Ardia, 1984.

Barański, Zigmunt. "La lezione esegetica di Inferno I: Allegoria, storia e letteratura nella Commedia". Picone 79-98.

Barberi-Squarotti, Giorgio. L'ombra di Argo - Studi sulla Commedia. Torino: Genesi, 1988.

Barbi, Mario. "Prefazione". Le opere di Dante. Firenze: Sansoni, 1921.

Barolini, Teodolinda. "Detheologizing Dante - For a 'New Formalism' in Dante Studies". Quaderni d'italianistica 10 (1989): 35-53.

Battaglia-Ricci, Lucia. Dante e la tradizione letteraria medievale. Pisa: Giardini Editori, 1982.

Blasucci, Luigi. "Linguaggio e stile della 'Divina Commedia". Il calendario del popolo, marzo 1965.

Boccaccio, Giovanni. Esposizioni sopra la Comedia di Dante. Ed. Giorgio Padoan. Tutte le opere di Giovanni Boccaccio. Ed. Vittore Branca. 6. Milano: Mondadori, 1965.

- Trattatello in laude de Dante. Giovanni Boccaccio. Opere in versi. Corbaccio. Trattatello in laude de Dante. Prose latine. Epistole. Ed. Pier Giorgio Ricci. Milano-Napoli: Ricciardi, 1965.

Bosco, Umberto, ed. Enciclopedia Dantesca. 6 voll. Roma: Istituto della Enciclopedia Italiana, 1970-1978.

Carugati, Giuliana. "Dante Mistico?". Quaderni d'italianistica 10 (1989): 237-50.

Cassell, Anthony. Lectura Dantis Americana, Inferno I. Philadelphia: U of Pennsylvania P, 1989.

Comparetti, Domenico. Virgil in the Middle Ages. London: Allen \&Unwin, 1966.

Consoli, Domenico, e Alessandro Ronconi. "Virgilio". Bosco 5.1030-44.

Contini, Gianfranco. Un'idea di Dante. Torino: Einaudi, 1976.

Corti, Maria. "Il modello analogico nel pensiero medievale e dantesco". Picone 11-20.

D'Andrea, Antonio. "L"'allegoria dei poeti'. Nota a Convivio 11.1". Picone 71-78.

D'Ovidio, Francesco. "L'Epistola a Cangrande". Rivista d' Italia 2 (1899): 3-33.

Dronke, Peter. The Medieval Poet and his World. Roma: Edizioni di Storia e Letteratura, 1984.

Eco, Umberto. Arte e bellezza nell'estetica medievale. Milano: Bompiani, 1987.

"L'Epistola X1ll, L'allegorismo medievale - Il simbolo modemo". Sugli Specchi e altri saggi. Milano: Bompiani, 1985.215-41.

.Il problema estetico in Tommaso d'Aquino. Milano: Bompiani, 1982.

Frankel, Margherita. "Juno among the Counterfeiters". Quaderni d' italianistica 10 (1989): 173-97.

Fubini, Mario. Il peccato di Ulisse e altri scritti danteschi. Milano-Napoli: Ricciardi, 1966.

Gilson, Ėtienne. The Spirit of Mediaeval Philosophy. New York: C. Scribner's Sons, 1940.

Grayson, Cecil. The World of Dante. Oxford: Clarendon P, 1980.

Green, Robert. "Dante's 'Allegory of the Poets' and the Medieval Theory of Poetic Fiction". Com. parative Literature 9 (1957): 18-128.

Hollander, Robert. Allegory in Dante's Comedy. Princeton: Princeton UP, 1969.

- Dante's Epistle to Cangrande. Ann Arbor: U of Michigan P, 1993.

-Il Virgilio dantesco: Tragedia nella 'Commedia'. Firenze: Olschki, 1983.

Studies in Dante. Ravenna: Longo, 1980.

Jenaro-MacLennan, Louis. The Trecento Commentaries on the Divina Commedia and the Epistle to Cangrande. Oxford: Clarendon P, 1974. 
Kelly, Henry Ansgar. Tragedy and Comedy from Dante to Pseudo-Dante. Berkeley-Los AngelesLondon: U of Califomia P, 1989.

Marigo, Aristide ed. Dante Alighieri. De Vulgari Eloquentia. Firenze: Le Monnier, 1968.

Mastrobuono, Antonio. Essays on Dante's Philosophy of History. Firenze: Olschki, 1979.

Mazzoni, Francesco. "Per l'Epistola a Cangrande". Studi in onore di Angelo Monteverdi. Modena: Società tipografica editrice modenese, 1959. 468-519.

Mazzotta, Giuseppe. Dante Poet of the Desert - History and Allegory in the Divine Comedy. Princeton: Princeton UP, 1979.

Mengaldo, Pier Vincenzo. "Stili". Bosco 5. 435-38.

Mineo, Nicolò. Profetismo e apocalittica in Dante. Catania: Università di Catania, 1968.

Minnis, Alastair J. Medieval Theory of Authorship. Philadelphia: U of Pennsylvania P, 1988.

Minnis, Alastair J., and Alexander Brian Scott. Medieval Theory and Criticism (c. 1100-c.1375). The Commentary-Tradition. Oxford: Clarendon P, 1988.

Moore, Edward. "The Genuiness of the Dedicatory Episte to Cangrande". Studies in Dante. Third Series. Oxford: Clarendon P, 1903. 284-369.

Nardi, Bruno. Dante e la cultura medievale. Bari: Laterza, 1983.

-Il punto sull'Epistola a Cangrande. Firenze: Le Monnier, 1960.

Pagliaro, Antonino. Ulisse: Ricerche semantiche sulla Divina Commedia. Firenze: G. D'Anna, 1967.

Paolazzi, Carlo. Dante e la 'Comedia' nel Trecento. Milano: Pubblicazioni della Università Cattolica del Sacro Cuore, 1989.

Paparelli, Gioacchino. Ideologia e poesia di Dante. Firenze: Olschki, 1975.

Pépin, Jean. "Allegoria". Bosco 1.151-65.

Petrocchi, Giorgio.Il Paradiso di Dante. Milano: Rizzoli, 1984.

Picone, Michelangelo, ed. Dante e le forme dell' allegoresi. Ravenna: Longo, 1987.

Pietrobono, Luigi. "L'Epistola a Cangrande". Giornale Dantesco 40 (1937): 1-51.

Risset, Jacqueline. Dante scrittore. Milano: Mondadori, 1984.

Schlosser-Magnino, Julius. La letteratura artistica. Firenze: La Nuova Italia, 1967.

Simonelli, Maria. "Convivio". Bosco 2.193-204.

Singleton, Charles. Commedia. Elements of Structure. Cambridge, Mass.: Harvard UP, 1954.

. "The Irreducible Dove". Comparative Literature 9 (1957): 129-35.

Tateo, Francesco. Questioni di poetica dantesca. Bari: Adriatica, 1972.

—. "La teoria degli stili e le poetiche umanistiche". Convivium 28 (1960): 141-64.

Thomas Aquinas. Summa Theologiae. Ed. Thomas Gilby, 61 voll. London: Blackfriars, Eyre and Spottiswoode, 1964-1981.

Villani, Francesco. Il comento al primo canto dell' Inferno. Ed. Giuseppe Cugnoni. Città di Castello: Cugnoni, 1896.

Zumthor, Paul. Langue et techniques poétiques à l'époque romane (XIle-XIlle siècles). Parigi: C. Klincksieck, 1963.

- Semiologia e poetica medievale. Milano: Feltrinelli, 1973. 